\title{
Riemann's theorem for quantum tilted rotors
}

\author{
G. Rosensteel and A.L. Goodman \\ Department of Physics, Tulane University, New Orleans, Louisiana 70118
}

\begin{abstract}
The angular momentum, angular velocity, Kelvin circulation, and vortex velocity vectors of a quantum Riemann rotor are proven to be either (1) aligned with a principal axis or (2) lie in a principal plane of the inertia ellipsoid. In the second case, the ratios of the components of the Kelvin circulation to the corresponding components of the angular momentum, and the ratios of the components of the angular velocity to those of the vortex velocity are analytic functions of the axes lengths.
\end{abstract}

PACS: 21.60.Ev, 21.60.Fw

Typeset Using REVTEX 
A classical Riemann rotor is a uniform density fluid with an ellipsoidal boundary and a velocity field that is a linear function of position. Riemann fluids model rotating stars and galaxies, [1.2] spinning gas clouds, [3] and rotating nuclei. [4.5] Since linear velocity fields span the dynamical continuum from rigid rotation to irrotational flow, the Riemann model is sufficiently general to model most collective rotational systems. The vector observables that measure the character of the rotation are the angular momentum $\vec{L}$ and the Kelvin circulation $\vec{C}$; 河 8 the vector variables conjugate to $\vec{L}$ and $\vec{C}$ are the angular velocity $\vec{\omega}$ and the vortex velocity $\vec{\lambda}$. Tilted nuclear rotors for which $\vec{\omega}$ is not aligned with a principal axis are a topic of continuing interest. [9 [17,?, 19, 20]

Recently, a quantum theory of Riemann rotors was formulated by simultaneous angular and vortex cranking of the nuclear mean field Hamiltonian,

$$
H_{\vec{\omega} \vec{\lambda}}=H_{0}-\vec{\omega} \cdot \vec{L}+\vec{\lambda} \cdot \vec{C},
$$

where the mean field is approximated by the anistropic oscillator potential,

$$
H_{0}=-\frac{\hbar^{2}}{2 m} \triangle+\frac{1}{2} m\left(\omega_{x}^{2} x^{2}+\omega_{y}^{2} y^{2}+\omega_{z}^{2} z^{2}\right) .
$$

Inglis's cranking formula determines the collective energy of an $A$-nucleon system, 21,22]

$$
T(\vec{\omega}, \vec{\lambda})=\sum_{p h} \frac{|\langle p|\vec{\omega} \cdot \vec{L}-\vec{\lambda} \cdot \vec{C}| h\rangle|^{2}}{\epsilon_{p}-\epsilon_{h}}
$$

Self-consistency of the mean field with the density distribution requires that

$$
\omega_{x} N_{x}=\omega_{y} N_{y}=\omega_{z} N_{z}
$$

where $N_{k}=\sum\left(n_{k}+1 / 2\right)$ denotes the total number of quanta in the $\mathrm{k}^{\text {th }}$ direction. The following semiclassical correspondence theorem has been established: 223] At self-consistency, the "Inglis" collective energy, Eq. (3), equals the classical value for the kinetic energy of a Riemann rotor, [1]

$T(\vec{\omega}, \vec{\lambda})=\frac{I_{0}}{4} \sum_{k=1}^{3}\left[\left(a_{i}^{2}+a_{j}^{2}\right)\left(\omega_{k}^{2}+\lambda_{k}^{2}\right)-4 a_{i} a_{j} \omega_{k} \lambda_{k}\right]$, 
where $i, j, k$ are cyclic, $a_{1}, a_{2}, a_{3}$ are the axes lengths of the inertia ellipsoid in units of $\mathrm{R}$, and the moment of inertia of a sphere of radius $\mathrm{R}$ and total mass $\mathrm{M}=\mathrm{mA}$ is $I_{0}=(2 / 5) M R^{2}$. If the nuclear volume is set equal to the spherical value $4 \pi R^{3} / 3$, then the product of the dimensionless axes lengths equals unity, $a_{1} a_{2} a_{3}=1$. Note that if the vortex velocity vanishes, then the self-consistent collective energy equals the rigid body energy, a well-known result. [24] In addition, the quantum expectations of the angular momentum and Kelvin circulation equal their classical Riemann rotor values, [1]

$$
\begin{aligned}
\left\langle L_{k}\right\rangle & =\frac{I_{0}}{2}\left[\left(a_{i}^{2}+a_{j}^{2}\right) \omega_{k}-2 a_{i} a_{j} \lambda_{k}\right] \\
\left\langle C_{k}\right\rangle & =\frac{I_{0}}{2}\left[2 a_{i} a_{j} \omega_{k}-\left(a_{i}^{2}+a_{j}^{2}\right) \lambda_{k}\right] .
\end{aligned}
$$

These expectations are given by derivatives of the kinetic energy with respect to the angular velocity and the vortex velocity [25]

$$
\left\langle L_{k}\right\rangle=\left(\frac{\partial T}{\partial \omega_{k}}\right),\left\langle C_{k}\right\rangle=-\left(\frac{\partial T}{\partial \lambda_{k}}\right) .
$$

The collective energy may be expressed as

$$
T(\vec{\omega}, \vec{\lambda})=\frac{1}{2}(\vec{\omega} \cdot\langle\vec{L}\rangle-\vec{\lambda} \cdot\langle\vec{C}\rangle)
$$

The energy in the rotating intrinsic frame for ordinary "Inglis" cranking of the angular velocity vector is minimized with respect to orientation when the angular velocity and angular momentum vectors are parallel. [11,12,20 Because the vortex velocity is independent of the angular velocity, minimization of the intrinsic energy $\tilde{E}(\vec{\omega}, \vec{\lambda})=\left\langle H_{\vec{\omega} \vec{\lambda}}\right\rangle$ with respect to the orientation of $\vec{\lambda}$ also requires that the vortex velocity and the Kelvin circulation vectors are parallel,

$$
\begin{aligned}
& \vec{\omega} \times\langle\vec{L}\rangle=0 \\
& \vec{\lambda} \times\langle\vec{C}\rangle=0 .
\end{aligned}
$$

This is proven by computing the change in the intrinsic energy when the orientation of the vortex velocity is shifted infinitesimally from $\vec{\lambda}$ to $\vec{\lambda}+\delta \vec{\lambda}=\vec{\lambda}+\epsilon \hat{n} \times \vec{\lambda}$, where $\hat{n}$ is an arbitrary unit vector, 


$$
\begin{aligned}
\tilde{E}(\vec{\omega}, \vec{\lambda}+\delta \vec{\lambda})-\tilde{E}(\vec{\omega}, \vec{\lambda}) & =\epsilon(\hat{n} \times \vec{\lambda}) \cdot\langle\vec{C}\rangle+O\left(\epsilon^{2}\right) \\
& =\epsilon \hat{n} \cdot(\vec{\lambda} \times\langle\vec{C}\rangle)+O\left(\epsilon^{2}\right)
\end{aligned}
$$

Hence, at equilibrium, $\hat{n} \cdot(\vec{\lambda} \times\langle\vec{C}\rangle)=0$ for all directions $\hat{n}$, or $\vec{\lambda} \times\langle\vec{C}\rangle=0$.

Riemann [26] proved in 1860 that the classical rotors in equilibrium fall into three classes:

1. Rigid rotors $(\lambda=0)$ which encompasses the Maclaurin spheroids and the Jacobi triaxial ellipsoids,

2. S-type ellipsoids for which the directions of $\vec{\omega}$ and $\vec{\lambda}$ are aligned with a principal axis, and

3. Tilted ellipsoids for which the directions of $\vec{\omega}$ and $\vec{\lambda}$ lie in a principal plane.

The case where $\vec{\omega}$ and/or $\vec{\lambda}$ do not lie in a principal plane is specifically excluded. It is remarkable that Riemann's theorem is also true for quantum cranked Riemann rotors. To prove the theorem, substitute the explicit formulae for the expectations of the angular momentum and Kelvin circulation, Eq. (6), into the parallelism conditions, Eqs. (9a, 9b). If the angular velocity vector $\vec{\omega}$ is neither aligned with a principal axis nor lies in a principal plane, then $\omega_{1}, \omega_{2}, \omega_{3}$ are all nonzero and the parallelism conditions constitute a set of six simultaneous equations in the three unknown ratios, $\lambda_{1} / \omega_{1}, \lambda_{2} / \omega_{2}, \lambda_{3} / \omega_{3}$. It can be shown that only four of these equations are independent. Therefore, this simultaneous system is overdetermined for the three unknown ratios. Since the assumption that all three components $\omega_{k}$ are nonzero implies a contradiction, one of the angular velocity components must vanish, say $\omega_{1}$. But, writing out the $y$-component of Eq. (9a), $0=\omega_{3}\left\langle L_{1}\right\rangle=-2 a_{2} a_{3} \omega_{3} \lambda_{1}$,

one concludes that $\lambda_{1}$ must also vanish. Hence, $\vec{\omega}$ and $\vec{\lambda}$ lie in a principal plane of the inertia ellipsoid. If $\omega_{2}$ and $\omega_{3}$ are both nonzero, then the parallelism conditions produce two independent equations in two unknown ratios whose solution is

$$
\begin{aligned}
& \lambda_{2} / \omega_{2}=\left(4 a_{1}^{2}-a_{2}^{2}+a_{3}^{2} \pm q\right) / 4 a_{1} a_{3} \\
& \lambda_{3} / \omega_{3}=\left(4 a_{1}^{2}+a_{2}^{2}-a_{3}^{2} \pm q\right) / 4 a_{1} a_{2},
\end{aligned}
$$


where

$$
q^{2}=\left(4 a_{1}^{2}-\left(a_{3}+a_{2}\right)^{2}\right)\left(4 a_{1}^{2}-\left(a_{3}-a_{2}\right)^{2}\right)
$$

If only one of the components of $\vec{\omega}$ is nonzero, then the angular velocity, vortex velocity, angular momentum, and Kelvin circulation vectors are all aligned with a single principal axis. The ratio $\lambda / \omega$ is undetermined for such an S-type ellipsoid. This completes the proof of Riemann's theorem.

Since $q$ cannot be imaginary, there are only three types of tilted rotors. Choosing an ordering of the axes lengths in the principal plane, say $a_{3} \geq a_{2}$, yields the following:

- Type I. $2 a_{1} \geq a_{3}+a_{2}$

- Type II. $2 a_{1} \leq a_{3}-a_{2}$ and $a_{2} \leq a_{1}$

- Type III. $2 a_{1} \leq a_{3}-a_{2}$ and $a_{1} \leq a_{2}$.

Type II and Type III tilted rotors are ultradeformed prolate-like solutions for which the ratio of the longest to the shortest axis is at least three to one. Type I solutions are tilted oblate-like rotors; these have been studied in the classical macroscopic approximation where the potential energy is a sum of the attractive surface energy plus the repulsive Coulomb potential. [5] 


\section{REFERENCES}

1 S. Chandrasekhar, Ellipsoidal Figures of Equilibrium, (Yale University Press, New Haven, 1969).

2 Norman R. Lebovitz, Ann. Rev. Astr. Astrophys. 5, 465 (1967).

$3 \quad$ F.J. Dyson, J. Math. Mech. 18, 91 (1968).

$4 \quad$ R.Y. Cusson, Nucl. Phys. A114, 289 (1968).

$5 \quad$ G. Rosensteel, Ann. Phys. (N.Y.) 186, 230 (1988).

6 O.L. Weaver, R.Y. Cusson, and L.C. Biedenharn, Ann. Phys. (N.Y.) 102, 493 (1976).

$7 \quad$ P. Gulshani and D.J. Rowe, Can. J. Phys. 54, 970 (1976).

$8 \quad$ G. Rosensteel and D.J. Rowe, Ann. Phys. (N.Y.) 96, 1 (1976).

$9 \quad$ H. Frisk and R. Bengtsson, Phys. Lett. B 196, 14 (1987).

10 Alan L. Goodman, Phys. Rev. C 45, 1649 (1992); Nucl. Phys. (to be published).

11 W. Nazarewicz and Z. Szymanski, Phys. Rev. C 45, 2771 (1992).

12 D.J. Thouless and J.G. Valatin, Nucl. Phys. 31, 211 (1962).

13 E.R. Marshalek, Nucl. Phys. A331, 429 (1979); in Proceedings of the Symposium on Contemporary Physics, Philadelphia, 1991 (unpublished).

14 I.N. Mikhailov and D. Janssen, Phys. Lett. B 72, 303 (1978); D. Janssen and I.N. Mikhailov, Nucl. Phys. A318, 390 (1979).

15 M.G. Vassanji and M. Harvey, Nucl. Phys. A344, 61 (1980).

16 A.K. Kerman and N. Onishi, Nucl. Phys. A361, 179 (1981).

17 F. Cuypers, Nucl. Phys. A468, 237 (1987).

18 A. Klein and M.G. Vassanji, Phys. Rev. Lett. 42, 436 (1979). 
19 F. Dönau, Nucl. Phys. A517, 125 (1990).

20 S. Frauendorf and T. Bengtsson, in Proceedings of the Workshop on Nuclear Structure in the Era of New Spectroscopy, Part B, Copenhagen, 1989 (unpublished).

21 D.R. Inglis, Phys. Rev. 96, 1059 (1954).

22 Peter Ring and Peter Schuck, The Nuclear Many-Body Problem, (Springer-Verlag, New York, 1980).

23 G. Rosensteel, Phys. Rev. C, in press.

24 Aage Bohr and Ben R. Mottelson, Nuclear Structure, (Benjamin, Reading, 1975) Vol. II.

25 R.P. Feynman, Phys. Rev. 56, 340 (1939).

26 B. Riemann, Abh. K. Ges. Wis. Göttingen 9, 3 (1860). 